\title{
Blood Flow Assessment in a Heart with Septal Defect based on Optical Flow Analysis of Magnetic Resonance Images
}

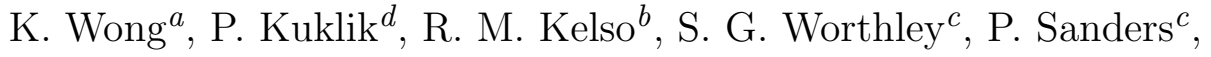 \\ J. Mazumdar ${ }^{a}$ and D. Abbott ${ }^{a}$ \\ ${ }^{a}$ School of Electrical and Electronic Engineering, University of Adelaide, SA 5005, Australia; \\ ${ }^{b}$ School of Mechanical Engineering, University of Adelaide, SA 5005, Australia; \\ ${ }^{c}$ School of Medicine, University of Adelaide, SA 5005, Australia; \\ ${ }^{d}$ Faculty of Physics, Warsaw University of Technology, 00-661, Poland;
}

\begin{abstract}
This study describes an application based on the optical flow algorithm to construct a $2 \mathrm{D}$ velocity field plot. The estimated velocity field is used to track the movement of blood in real time. This methodology has been applied to medical images to quantify blood flow turbulence in the right atrium of the heart. Blood intensity fields that are obtained from clinical MRI scan sequences can be analyzed using this method. Septal defects and other heart diseases can be assessed for degrees of abnormality and post-surgical success can be evaluated. We have developed this technique specifically for characterizing the turbulence generated due to such heart abnormalities. The degree of turbulence and fluid shear stress can be determined from the measured flow field. The cardio dynamics information that is based on flow analysis and visualization of blood offers potential for the detection and quantification of myocardial malfunctioning.
\end{abstract}

Keywords: Atrial septal defect, Magnetic resonance imaging, Optical flow

\section{INTRODUCTION}

Septal defect is an abnormality of the heart and can be categorized into ventricular septal defect (VSD), and atrial septal defect (ASD). For both cases, oxygenated blood is forced from the left to the right side of the heart through a hole in the septum. This results in excessive blood passing into the lungs (via the pulmonary artery) and too little to the body tissues (via the aorta), which results in insufficient distribution of oxygen to the other parts of the body.

Ventricular septal defect has a $0.15 \%$ chance of occurrence before birth. A small defect has a minor or no effect on health, but a large ventricular hole can result in heart failure six to eight weeks after birth. With large atrial defects, heart failure may develop at a later stage. The existing method for diagnosis is based on detection of a heart murmur by a stethoscope. This occurrence is the result of the abnormal heart sounds made by turbulent blood flow. This is then followed by a chest X-ray and echo-cardiogram scans (ECG). ${ }^{1}$ Atrial or ventricular defects may be observed from these medical images. Flow visualization may be achieved using pulsed or continuous wave Doppler ultrasound. Although visualization of the defected cardiac structure using X-rays, echocardiogram or ultrasound methods are limited in clarity and resolution, they suffice to provide a clinical diagnosis of the sick patient.

Until recently, the only way to seal the defect was by open-heart surgery. The use of Cardiac Catheterisation (CC) is a minimally invasive technique which involves inserting a catheter into the chambers of the heart through one of the arteries passing through the groin of a human. ${ }^{2}$ A method known as the key-hole procedure in which a septal occluder is inserted into the femoral artery via a tube and directed through to the heart, is now commonly used. At the defective cardiac wall, the device that is constructed of a wire mesh opens like an umbrella and covers the hole. After a few minutes, a blood clot forms on the wire discs of the occluder making a secure seal. Over time, the cardiac tissue will grow to surround the septal occluder.

Further author information: (Send correspondence to K. Wong and D. Abbott)

K. Wong: E-mail: kwong@eleceng.adelaide.edu.au, Telephone: +61 883036296

D. Abbott: E-mail: dabbott@eleceng.adelaide.edu.au, Telephone: +61 883034360

Biomedical Applications of Micro- and Nanoengineering III, edited by Dan V. Nicolau, Proc. of SPIE Vol. 6416, 64160L, (2006) · 1605-7422/06/\$15 - doi: 10.1117/12.696691 
As an alternative to the mentioned diagnostic technique, we have applied the optical flow ${ }^{3}$ (OF) scheme to MRI images and performed flow quantification to analyze, in particular, the blood movement in the right atrium based on the measured flow field. The intensity-based MRI images can be assessed for blood movements based on the optical flow evaluation. As such, this technique offers potential for the non-invasive flow visualization in cardiac structures after the septal occluder implant for a beating heart that changes its spatial position with time.

The optical flow technique is useful for evaluating blood flow abnormalities in the heart chambers from MRI images. The global estimation of flow velocity vector fields across the whole image provides useful information on turbulence in pathology within the heart chamber. Therefore, optical flow evaluation of MRI images is able to provide flow field measurement within the relevant pathology. This type of approach has been used in image processing and computer vision for more than two decades, but has found few applications in medical imaging to date. Previously, the OF scheme has been used for only one experimental flow study, ${ }^{4}$ and compared favourably with results obtained from the well established Particle Image Velocimetry (PIV) technique. The scheme includes a specific enhancement that is able to provide accurate dense motion fields of up to one vector per pixel.

The application of the optical flow technique to the MRI image grid, based on the reconstruction of slices, allows non-linear flow velocity field on a two-dimensional velocity grid to be measured. Moreover, the construction of vorticity, shear strain and shear stress distributions, based on the velocity field, can be used to provide insights into the behavior of the flow. A feature of this method is the computation and presentation of these flow features temporally in $2 \mathrm{D}$.

This article is organized as follows: In the first section, we examine the conventional imaging techniques that are used for motion analysis. Next, we discuss the methodology and experimental procedures. In the subsequent sections, we provide flow results and establish a form of flow quantification based on the 2D analysis. Finally, the results are discussed and conclusions are made.

\section{LITERATURE REVIEW}

The echocardiogram is an ultrasound scan of the heart. Using standard ultrasound scans, two-dimensional slices of the heart can be imaged that can be used in flow visualization. In addition to creating scans of the cardiovascular system, the echocardiogram can also produce an assessment of the velocity of blood and cardiac tissue at any arbitrary point using pulsed or continuous wave Doppler ultrasound. This allows assessment of cardiac valve areas and function, any abnormal communications between the left and right side of the heart, any leaking of blood through the valves (valvular regurgitation), and calculation of the cardiac output as well as the ejection fraction.

Ultrasound is sensitive to blood flow, which makes it potentially applicable to flow visualization. However, this technique is currently used only as a qualitative and not quantitative modality. Contrast agents exist as a suspension of microscopic gas bubbles, which are used to enhance ultrasound performance. Ultrasound is, however, an inexpensive alternative to MRI, PET and CT modalities.

The use of optical flow for image scans in conjunction with ultrasound, CT, ${ }^{5}$ as well as tagged MRI, ${ }^{6}$ and other 3D imaging methods has been suggested previously. Efforts have been made to use ultrasound data for motion analysis, which include the intensity-based optical flow method. However, applying optical flow analysis to ultrasound and echocardiography is limited by local area distortion and poor resolution. ${ }^{7}$

Note that OF analysis has been performed on tagged MRI images before, but most of the analysis are on the movement of the arterial and cardiac walls ${ }^{1,2,8}$ and not on the blood flow itself. Currently available assessments quantifying blood flow have not been able to resolve the vortices that exist during flow. Such methods have been based on ultrasound technology providing flow rate quantification.

The assessment of flow in the blood has been performed on echocardiograms ${ }^{1,9}$ as well. A sequence of grayscale B-mode images can be acquired from a human heart during a cardiac cycle for this purpose. However the accuracy of the motion estimation is limited by the local image distortion.

Therefore, the application of OF techniques to medical images offers the potential for velocity measurement to become a commonly accessible technology. The determination of flow velocities is based on the intensity shifts 
of the pixels using the OF algorithm. The assessment of flow is accurate provided that a good time resolution is present. However, the number of phases per cardiac cycle will be dependent on the capabilities and specifications of the particular medical imaging device used.

\section{METHODOLOGY}

This section describes the nature and framework of our experiment, scan procedures and method of data analysis. Various computational techniques that are vital to the segmentation of cardiac chamber and flow analysis are briefly mentioned. It also describes the output of the software that is used in flow visualisation.

\subsection{Subject}

A male subject aged 19 years at the time of this experiment volunteered for this study and received approval from the institutional ethics committee. The subject had a septal defect in the partition between the left and right atria and was assessed before and after implantation of the septal occluder.

\subsection{Clinical diagnosis}

In our clinical examination, the right atrium was selected as the region of analysis since the changes in the intensity of turbulence are more easily detected than in the other chambers. This is because the turbulence is more prominent in the right atrium due to injection of blood from the left atrium through the septal defect. This region of interest must be segmented for flow visualisation and so active contouring based on the Kass-snake algorithm ${ }^{8}$ is carried out.

We have analysed images of the heart using motion estimation algorithms. In this particular example, the Horn-Schunck optical flow ${ }^{3}$ scheme on the intensity-based MRI images of the human heart was chosen. The measurement of velocity in blood flows through image sequences is represented using a velocity vector field (quiver arrow plot). More specific details are set out below, including the quantification of parameters indicating the intensity of turbulence and the analysis of $2 \mathrm{D}$ image slices.

\subsection{MRI Scan Procedure}

The human subject was scanned based on standard MRI procedure to output 9 slices of the cardiac structure at $6 \mathrm{~mm}$ resolution along the axial plane. The time resolution of the scan was 25 phases $(T=0$ to 24$)$ per cardiac cycle. Based on the scans, two sets of 225 image slices in bitmap format at 123 by 140 and 156 by 192 pixels resolution for the prior and post operation scans respectively were exported for optical flow analysis.

One of the slices showing the sectioning of the right and left atriums was selected for visualisation and examination. The use of MRI scans for flow visualization is unlimited by opacity of the structure and its motion. Movement of cardiac tissues is effectively excluded by performing contour segmentation on the cardiac walls.

\section{DATA ANALYSIS}

A computer application based on the proposed methodology is completed with important working functions to analyze flow from intensity-based MRI images. We can input medical images into this application and perform flow quantification by establishing blood motion vectors in the plane of the image. The intensity of turbulence and fluid shear strain can be estimated from the measured flow field. Parameters such as the level of vorticity (ROT), shear strain (SS) and normal strain (NS) can be been quantified and examined.

The plotting of arrows and usage of pixel intensity to represent vortices, shear strain and shear stress values for each interrogation window (with size being adjusted based on the number of layers of arrow contours sampled), as well as the construction of three-dimensional flow patterns is suggested. The statistical examination of these flow characteristics is developed after that. The model will allow quantification of flow in the cardiac chamber in real time. 


\subsection{Active Contour Segmentation}

The cardiac wall is segmented by placement of a $2 \mathrm{D}$ contour that forms a "balloon" within the cardiac chamber. The migration of balloon nodes from their origins onto the intense wall region is executed based on an energy minimisation algorithm. ${ }^{2,8}$ The initial curve can be anywhere in the image, and interior contours are automatically detected.

\subsection{Numerical Formulations}

We performed statistical quantification of the flow field in the aorta by examining the vorticity, shear strain and normal strain of the blood. These parameters ${ }^{10}$ are computed from the velocity gradients provided by the optical flow scheme. Based on the velocity contour of a pixel of interest denoted as $i$, where $N$ represents the number of layers of contour within the sampling frame, the flow characteristics can be numerically determined:

Vorticity

$$
\operatorname{ROT}(N)=\sum_{n=1}^{N} \frac{V_{x}(i+n, j)-V_{x}(i-n, j)}{2 n \Delta y}-\frac{V_{y}(i, j+n)-V_{y}(i, j-n)}{2 n \Delta x}
$$

Shear Strain

$$
\mathrm{SS}(N)=\sum_{n=1}^{N} \frac{V_{x}(i+n, j)-V_{x}(i-n, j)}{2 n \Delta y}+\frac{V_{y}(i, j+n)+V_{y}(i, j-n)}{2 n \Delta x}
$$

Normal Strain

$$
\mathrm{NS}(N)=\sum_{n=1}^{N} \frac{-V_{x}(i+n, j)+V_{x}(i-n, j)}{2 n \Delta y}+\frac{-V_{y}(i, j+n)+V_{y}(i, j-n)}{2 n \Delta x} ;
$$

Typically, as $N$ increases, the accuracy of flow parameter determination improves. In our experiments, a neighborhood of one layer $(N=1)$ has been introduced into the calculations at the data analysis stage.

\section{RESULTS}

In this section, we discuss the flow patterns of blood in the right atrium by analysing the 1) vorticity (ROT), 2) shear strain (SS), and 3) normal strain (NS) of each pixel or group of pixels in the image. It is proposed that the turbulence generated by an atrial or ventricular septal defect will be significantly higher than the turbulence present within a healthy pathology. An indication of the intensity of turbulence can be provided by generating histograms depicting the counts of pixels on the vertical axis with their respective values of ROT, SS, and NS in the horizontal axis.

The means of the histograms for all swirl and strain parameters is calculated. The direction of vortices and strains is disregarded and only the absolute values of these parameters contributed to the calculation of the mean value. The means of absolute ROT, SS, and NS are indicators for the global estimation of vorticity, shear and normal strains in the region of interest. The measure of the spread or dispersion within the set of pixel counts for a range of ROT, SS and NS values also provides information on the inherent level of the measured parameter values. A higher variance of a particular flow parameter denotes a greater intensity within the area of interest from which the counts of pixels pertaining to that parameter value are based on. In this paper, the means and variances of the [ROT, SS and NS] histograms are denoted as $\left[\mu_{1}, \mu_{2}, \mu_{3}\right]$ and $\left[\sigma_{1}^{2}, \sigma_{2}^{2}, \sigma_{3}^{2}\right]$ respectively.

The global estimation of flow velocity vector fields over the whole image provides useful information on the presence of vortices within the pathology that can be used to assess the intensity of turbulence in the atrium. 


\subsection{Flow Analysis based on 2D Image Slices}

MRI scans of the heart for six phases using the short axis orientation shows the post contraction state of the left atrium and ejection of blood into the right atrium for prior and post septal occluder insertion (Appendix A and B). Based on each of the image, the velocity vector plot with its corresponding density histogram of the flow characteristics at six phases is computed. The top bar pertains to the vorticity histogram, followed by the shear strain and normal strain histograms at the bottom. From the magnitude of the histogram bins, we are able to deduce the degree of the localised blood rotation and strain. Normalisation of the histograms is performed by setting the sum of all bin counts in each histogram chart to 3500 for each of the images. The number of bins is set at 35. Normalisation is a necessity as the size of the region of measurement is different for every image.

Figure 1 presents velocity vector fields at six phases of one heart cycle from time phases, $T=18$ to 23 during the event of sudden left atrium contraction and forcing of blood through the hole at the partition between the right atrium and left atrium. The vector field shows that the blood flow near the entrance of the septal defect contains turbulent eddies that are distributed across the entire flow area.

The most significant features present in the velocity fields of Figure 1 are the wave-like features at phases $T=21$ through to $T=23$. These features appear to show a series of large-scale vortices radiating out from the septal defect on the right side (as appears in the image). These are most likely formed by the sudden jet of fluid from the defect. The readjustment in the distribution of the histograms beginning at these observed phases is the result of the increased blood shear interface in between the atrial and the jet flow through the defect. Therefore, the counts for the absolute values greater than zero are relatively greater. The flow interaction causes shearing and stresses of the blood cells and this can increase the risk of thrombosis. Detection of this phenomenon can be further supported by the quantification of shear and normal strain changes of the global region of the chamber.

Figure 2 shows the histograms of flow within the atrium after occluder insertion. It may be observed that there is reduced turbulence due to the fact that the amount of leakage that occurs through the septal defect is minimised by the septal occluder. Vorticity, shear strain and normal strains are present in the fluid when there is turbulence. The results show that generally, there is a lower turbulence at all phases. The turbulence structure is similar for $T=18$ to 20 . There is significantly lower turbulence for $T=19$ to 23 and no evidence of lateral jet from the defect can be observed.

Figure 3 shows the changes in the means $\mu_{1}, \mu_{2}$ and $\mu_{3}$ pertaining to ROT, SS and NS based on the time phases from $T=0$ to 23 for prior and post operation. The relative magnitude of the vorticity, shear strain and stress strain for the two sets of graphs is demonstrated. This provides indication of whether these parameters are affected before and after operation.

Figure 4 shows the changes in the variances $\sigma_{1}^{2}, \sigma_{2}^{2}$ and $\sigma_{3}^{2}$ pertaining to ROT, SS and NS based on the time phases from $T=0$ to 23 for prior and post operation. These graphs can be used to assess the redistribution of flow structures as the blood swirls within the atrium. A higher variance corresponds to a greater global magnitude for flow turbulence or blood strain within the cardiac chamber.

It can be observed that $\left[\mu_{1}, \mu_{2}, \mu_{3}\right]$ and $\left[\sigma_{1}^{2}, \sigma_{2}^{2}, \sigma_{3}^{2}\right]$ for the flow prior to operation is relatively higher than that for measurements of flow taken after operation. The magnitudes of the mean and variance for ROT and NS have small difference for prior and post occluder insertion. This demonstrates that an increase in localised fluid rotation corresponds to an increase in the normal strain of the fluid. This can be explained by the occurrence of swirling that is measured by the vorticity which disturbs the stationary state of flow and adds strain and stresses to the blood. The shear strain however, takes a larger increase as compared to the normal strain. There is an increase in the mean and variance of SS before and after the occluder insertion from $T=19$ through to $T=21$. After sealing the septal defect, the intensity of swirl is minimised by the blockage of the occluder.

\section{DISCUSSION}

The examination of MRI scans prior to and post septal occluder insertion revealed differences in intensity of the blood shear and normal strains in the heart. This information indicates the degree of abormality in the cardiac structure and provide clinical diagnosis of the septal defect. An appropriate medical treatment may be proposed based on the quantitative assessment of the problem. 

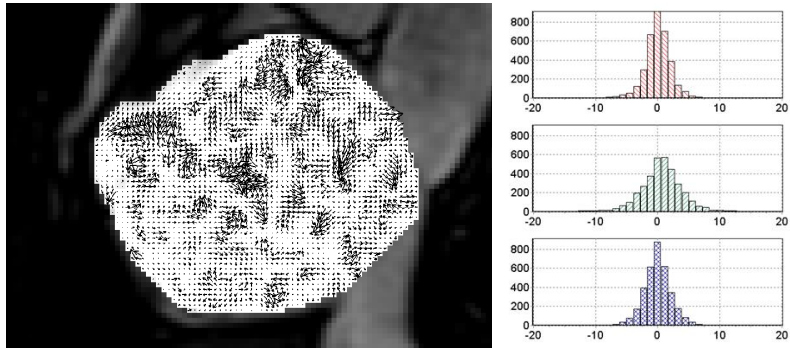

(a) $T=18$

Means of absolute values: $\mu_{1}=1.58, \mu_{2}=2.66, \mu_{3}=1.7$ Variances of charts: $\sigma_{1}^{2}=4.85, \sigma_{2}^{2}=12.61, \sigma_{3}^{2}=5.32$
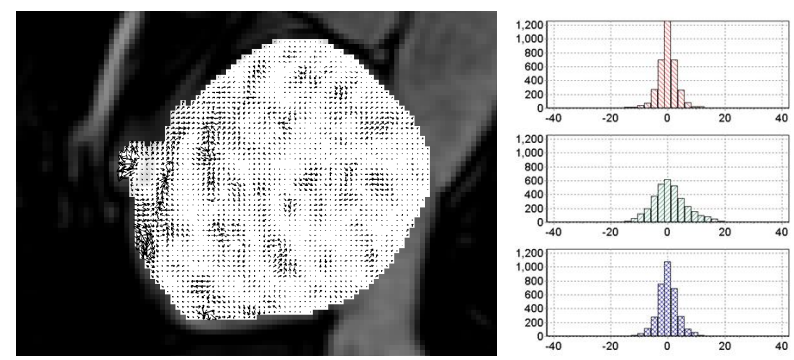

(c) $T=20$

Means of absolute values: $\mu_{1}=2.58, \mu_{2}=4.94, \mu_{3}=2.9$ Variances of charts: $\sigma_{1}^{2}=14.27, \sigma_{2}^{2}=44.31, \sigma_{3}^{2}=17.17$
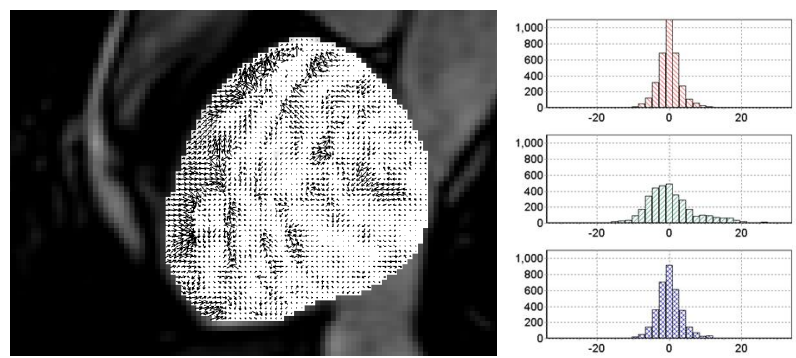

(e) $T=22$

Means of absolute values: $\mu_{1}=2.34, \mu_{2}=5.31, \mu_{3}=2.67$

Variances of charts: $\sigma_{1}^{2}=10.76, \sigma_{2}^{2}=52.19, \sigma_{3}^{2}=13.5$
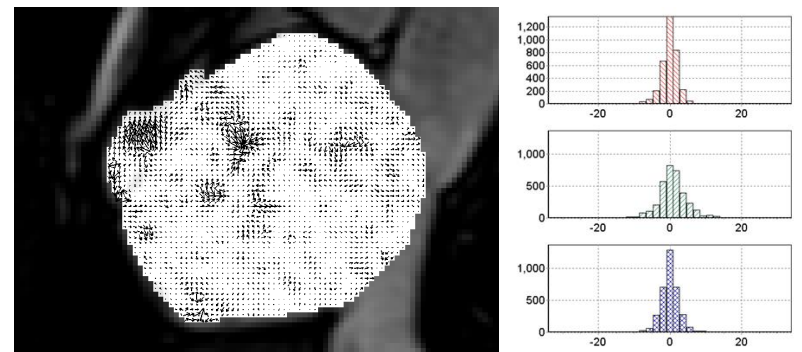

(b) $T=19$

Means of absolute values: $\mu_{1}=1.74, \mu_{2}=3.21, \mu_{3}=2.06$

Variances of charts: $\sigma_{1}^{2}=6.1, \sigma_{2}^{2}=20.51, \sigma_{3}^{2}=10.04$
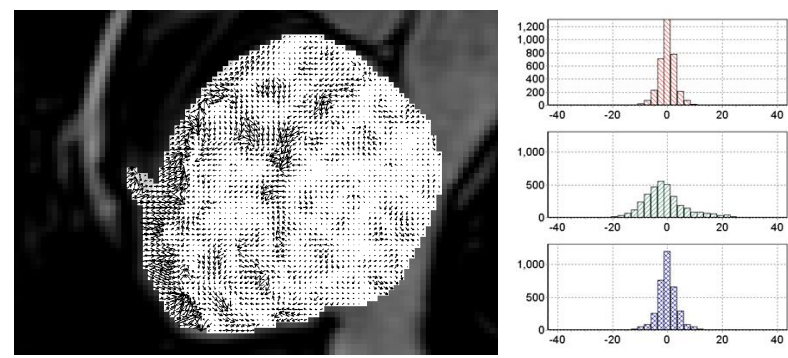

(d) $T=21$

Means of absolute values: $\mu_{1}=2.35, \mu_{2}=6.49, \mu_{3}=2.68$ Variances of charts: $\sigma_{1}^{2}=10.83, \sigma_{2}^{2}=77.85, \sigma_{3}^{2}=14.31$
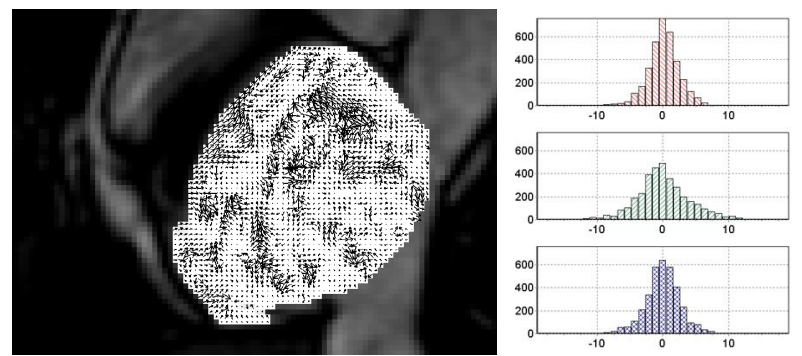

(f) $T=23$

Means of absolute values: $\mu_{1}=1.85, \mu_{2}=3.03, \mu_{3}=2.03$

Variances of charts: $\sigma_{1}^{2}=6.15, \sigma_{2}^{2}=16.25, \sigma_{3}^{2}=7.59$

Figure 1. Velocity field of right atrium (prior to septal occluder insertion) are shown by velocity vector plots superimposed on MRI images at six selected time phases ( $T=18$ to 23 ) of one cardiac cycle. Their respective flow density plots are presented on the right. The vorticity, shear strain and normal strain functions are shown starting from the top to the bottom charts 

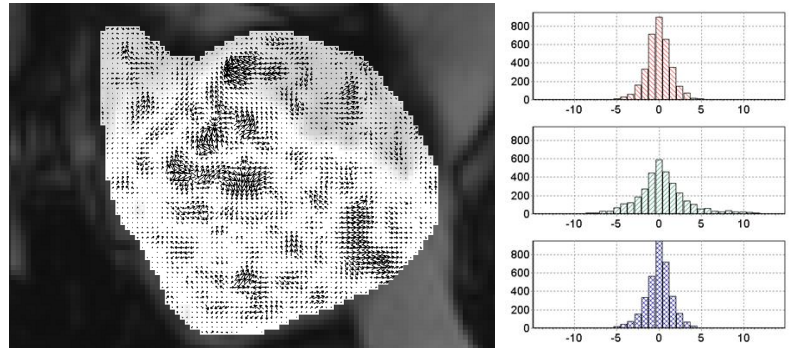

(a) $T=18$

Means of absolute values: $\mu_{1}=1.14, \mu_{2}=2.32, \mu_{3}=1.19$

Variances of charts: $\sigma_{1}^{2}=2.33, \sigma_{2}^{2}=10.97, \sigma_{3}^{2}=2.79$
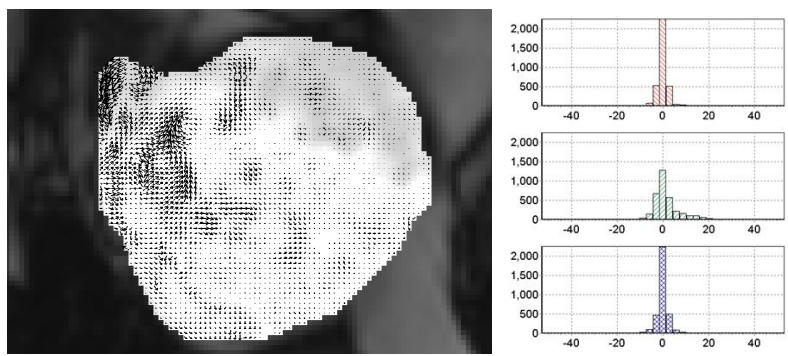

(c) $T=20$

Means of absolute values: $\mu_{1}=1.65, \mu_{2}=4.21, \mu_{3}=1.74$

Variances of charts: $\sigma_{1}^{2}=7.63, \sigma_{2}^{2}=48.71, \sigma_{3}^{2}=8.47$
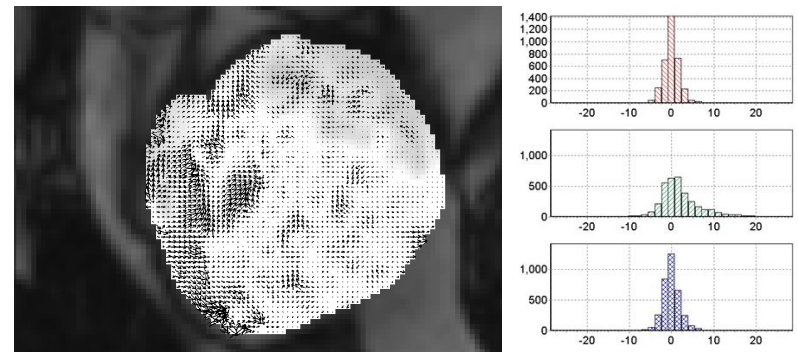

(e) $T=22$

Means of absolute values: $\mu_{1}=1.44, \mu_{2}=3.68, \mu_{3}=1.62$

Variances of charts: $\sigma_{1}^{2}=4.16, \sigma_{2}^{2}=24.23, \sigma_{3}^{2}=5.52$
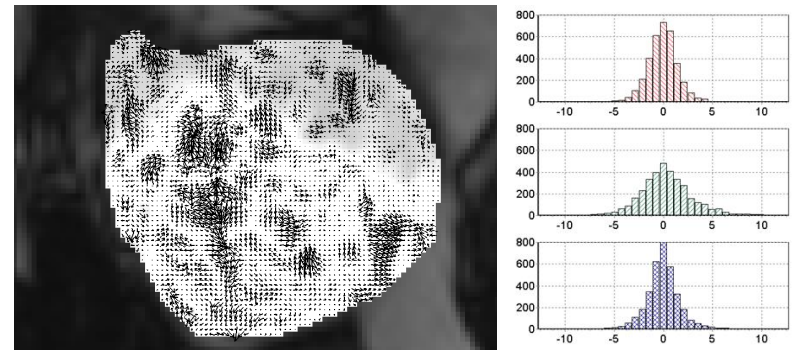

(b) $T=19$

Means of absolute values: $\mu_{1}=1.16, \mu_{2}=2.03, \mu_{3}=1.26$

Variances of charts: $\sigma_{1}^{2}=2.34, \sigma_{2}^{2}=7.42, \sigma_{3}^{2}=3.27$
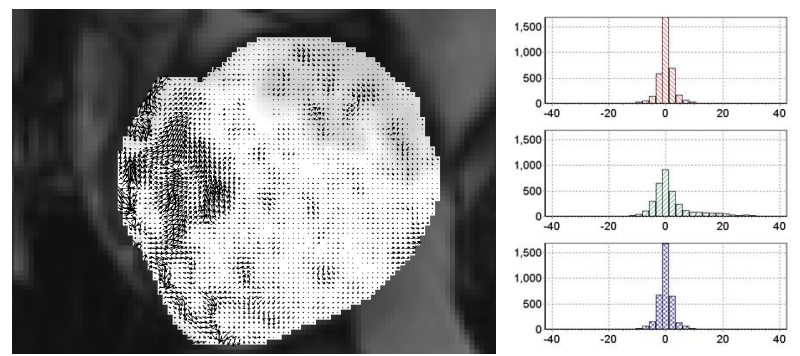

(d) $T=21$

Means of absolute values: $\mu_{1}=1.99, \mu_{2}=5.29, \mu_{3}=2$

Variances of charts: $\sigma_{1}^{2}=9.31, \sigma_{2}^{2}=66.83, \sigma_{3}^{2}=10.3$
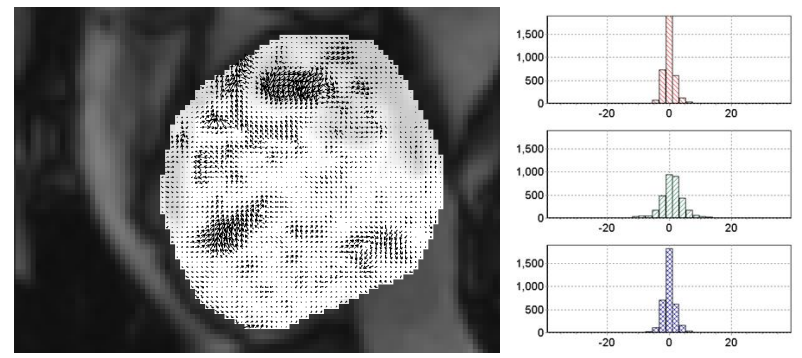

(f) $T=23$

Means of absolute values: $\mu_{1}=1.36, \mu_{2}=3.43, \mu_{3}=1.47$

Variances of charts: $\sigma_{1}^{2}=3.7, \sigma_{2}^{2}=29.49, \sigma_{3}^{2}=4.3$

Figure 2. Velocity field of right atrium (post septal occluder insertion) are shown by velocity vector plots superimposed on MRI images at six selected time phases $(T=18$ to 23$)$ of one cardiac cycle. Their respective flow density plots are presented on the right. The vorticity, shear strain and normal strain functions are shown starting from the top to the bottom charts 


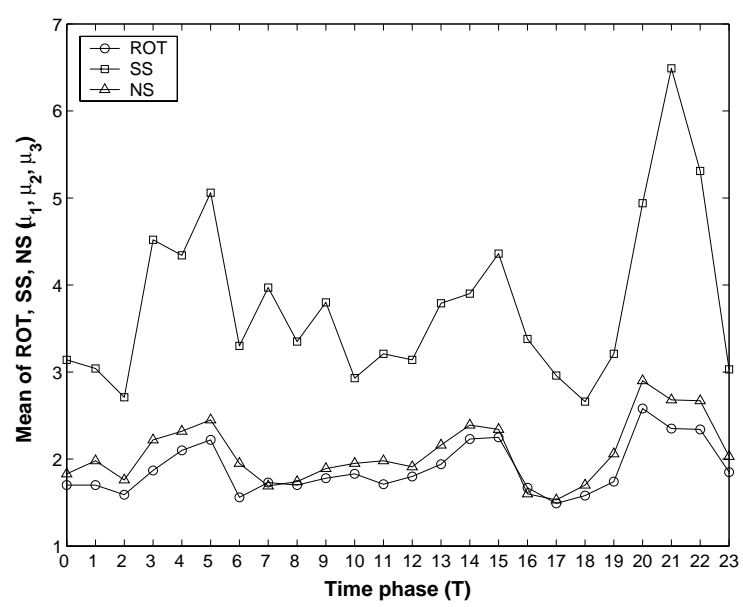

(a) Pre-occluder insertion

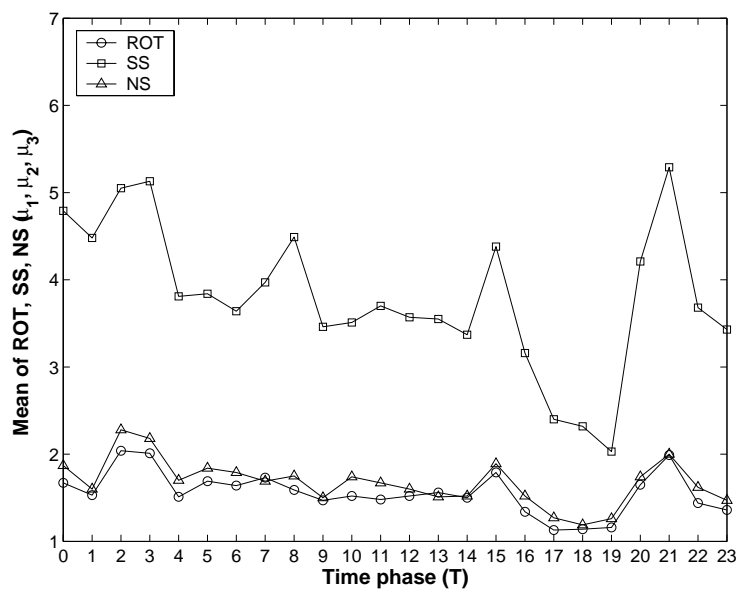

(b) Post-occluder insertion

Figure 3. Means $\left(\mu_{1}, \mu_{2}\right.$ and $\left.\mu_{3}\right)$ of ROT, SS and NS as a function of time phase $(T)$.

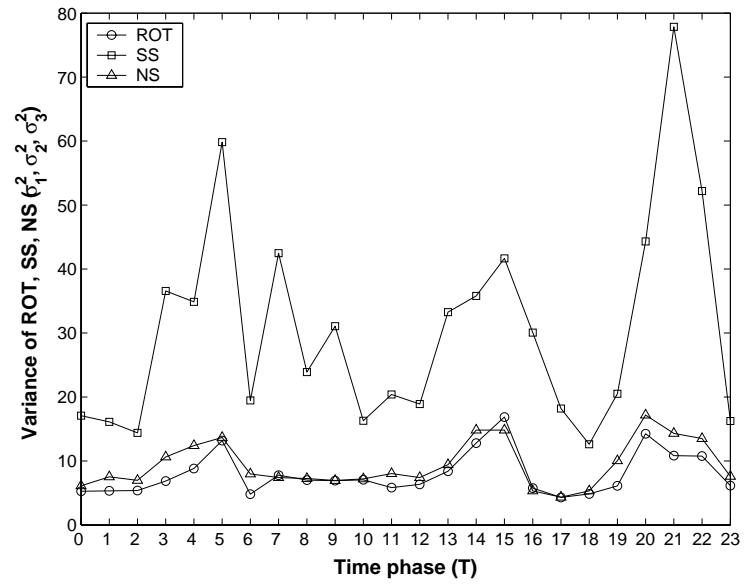

(a) Pre-occluder insertion

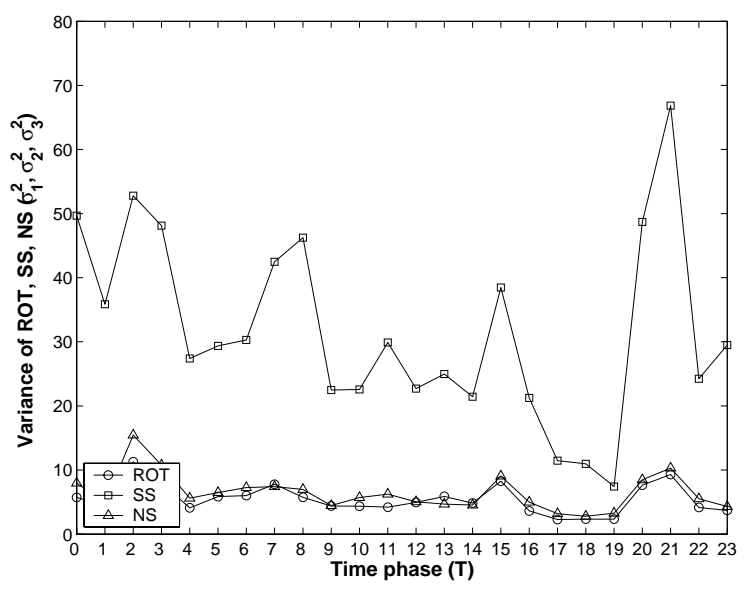

(b) Post-occluder insertion

Figure 4. Variances $\left(\sigma_{1}^{2}, \sigma_{2}^{2}\right.$ and $\left.\sigma_{3}^{2}\right)$ of ROT, SS and NS as a function of time phase $(T)$. 
Because the image sizes for the prior and post operation scans are different, we need to set the area under the histogram graph (sum of number of counts for every bin) for each of the ROT, SS, and NS histograms as a constant. This is performed for both sets of images to attain a more controlled comparison. Since the total number of pixels measured is now normalised and set as a constant, if the counts of vorticity and strain pertaining to bins of very small values exceed that of the counts at other larger absolute values significantly, it means that within the region of measurement, the flow is still dominated by stationary groups of pixels. Comparatively, if the counts of the non-zero flow are lower, the level of flow swirls is relatively higher as there are more counts of vorticity and strains with non-zero absolute values.

Generally, at a particular time instance, if the vector field demonstrated that most regions of blood in the atrium is stagnant or experiencing a laminar flow, the mean vorticity should be close to zero. In the case of the abnormal heart, it is likely that the mean vorticity and strain will shift away from zero, and broaden the distribution of the histogram. Therefore, as demonstrated, the flow vorticity, shear strain and normal strain counts for pre-operation are more significant than those for the post-operation. This is evidence that the methodology is successful in assessing the relative intensity of turbulence before and after insertion of the septal occluder.

The display of information using histograms of vorticity, shear and normal strains provides a clear overview of the swirl and stress present within the blood flow. The variation in the level of these parameters at each phase can be used to determine the degree of abnormality in the cardiac structure. A sudden change in value of the flow parameter depicts a flow event at a particular time phase.

At the preliminary stage of this research, we present flow in the $2 \mathrm{D}$ plane. The quantification of vorticity, shear stress and shear strain on 2D slices is an approximation of the 3D model. However it suffices to assess the level of turbulence for blood flow at this stage. The presence of vortices in more than one orthogonal plane provides a better indication of the 3D structure and can more effectively reveal flow phenomena that may have implications in cardiac operations. In addition, we are only able to assess just one case of ASD. This may not provide sufficient justification that the proposed application has been successful in determining the decrease turbulence level for blood in the right atrium before and after septal occluder insertion.

The use of other human subjects to act as controls for this experiment is difficult as every individual has a different heart structure and therefore the degree of turbulence will have been biased even under a controlled environment. The experiments are only possible for measurements of flow in the same heart before and after an operation.

\section{CONCLUSIONS}

There are currently no commercial systems available for the assessment of blood stress and strain to the best of our knowledge. Therefore, we have developed measurement-based software specially for analysing flow structures within pathology. The new methodology is based on the optical flow analysis of MRI scans and provision of the flow characteristics by analysing the flow vectors at every image pixel.

We have obtained results for a heart with a septal defect (prior and post operation). The new software has proved that, based on the calculations of vorticity, shear strain and normal strain, it is possible to detect the intensity of turbulence and therefore determine the degree of septal defect, and provide some indication of the success of the septal occluder insertion. The software may also be used to examine the growth of cardiac tissue into the mesh of the occluder. As it provides quantitative as well as qualitative data for this flow, the software can be of great benefit to surgeons for assessing their surgical success.

The method has been tested successfully for an abnormal pathology and a more quantitative diagnosis to add to the qualitative information has been obtained. The application may potentially be incorporated into MRI visualisation modelling and display for such a purpose.

\section{ACKNOWLEDGEMENTS}

We thank the medical team at the Royal Adelaide Hospital and Diana Pilkington at the Wakefield Hospital for assistance in the scanning and supply of MRI images. 


\section{REFERENCES}

1. M. Sühling, M. Arigovindan, C. Jansen, P. Hunziker, and M. Unser, "Myocardial motion analysis and visualization from echocardiograms," SPIE Int. Symp. Med. Imag. MI'03, pp. 306-313, 2003.

2. S. S. Jasjit, "Computer vision, pattern recognition and image processing in left ventricle segmentation: The last 50 years," Pattern Anal. Appl. 3(3), pp. 209-242, 2000.

3. B. K. P. Horn and B. G. Schunck, "Determing optical-flow," Artificial Intelligence 17 (1-3), pp. 185-203, 1981.

4. T. Corpetti, D. Heitz, G. Arroyo, E. Mémin, and A. Santa-Cruz, "Fluid experimental flow estimation based on an optical-flow scheme," Experiments in Fluids 40, pp. 80-97, 2006.

5. N. Weng, Y.-H. Yang, and R. Pierson, "Three-dimensional surface reconstruction using optical flow for medical imaging," IEEE Trans. Med. Imaging 16, p. 630, 1997.

6. K. W. Moser, J. G. Georgiadis, and R. O. Buckius, "On the use of optical flow methods with spin-tagging magnetic resonance imaging," Ann. Biomed. Eng. 29, p. 9, 2001.

7. V. Behar, D. Adam, and P. Lysyansky, "Improving motion estimation by accounting for local image distortion," Ultrasonics 43(1), pp. 57-65, 2004.

8. L. D. Cohen, "On active contour models and balloons," Computer Vision, Graphics, and Image Processing 53(2), pp. 211-218, 1991.

9. M. Sühling, M. Arigovindan, C. Jansen, and et al., "Myocardial motion analysis from B-mode echocardiograms," IEEE Trans. on Img. Proc. 14(4), pp. 525-536, 2005.

10. M. Raffel, C. Willert, and J. Kompenhans, Particle Image Velocimetry, Springer-Verlag Berlin Heidelberg New York, 1998. 

(PRIOR TO SEPTAL OCCLUDER INSERTION)

Observation of blood ejected through ASD of heart

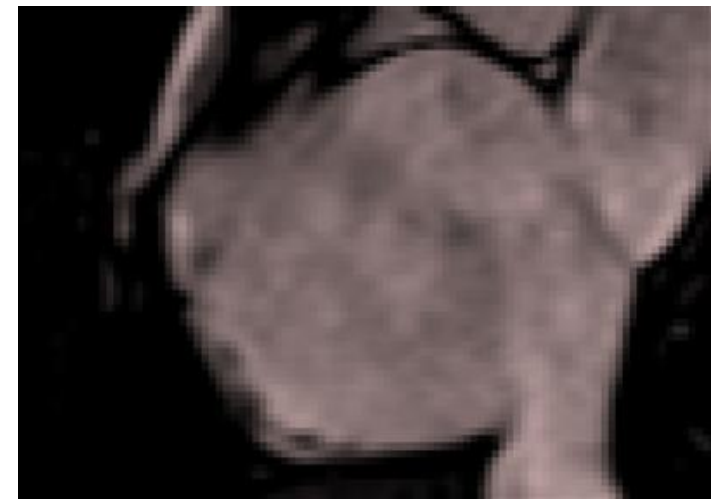

(a) $T=18$

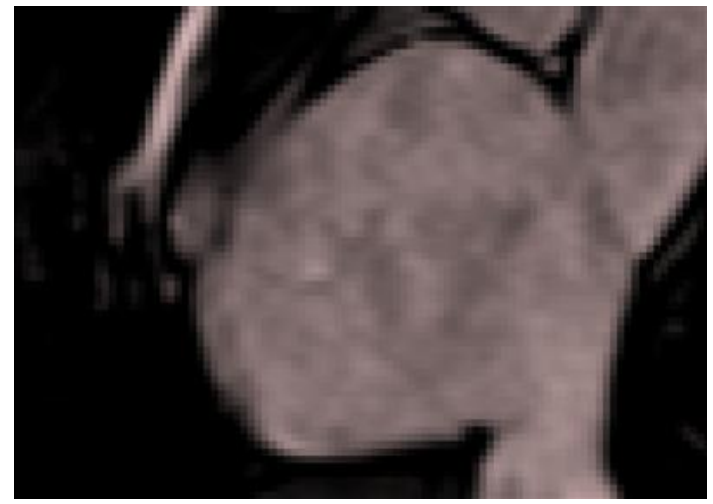

(c) $T=20$

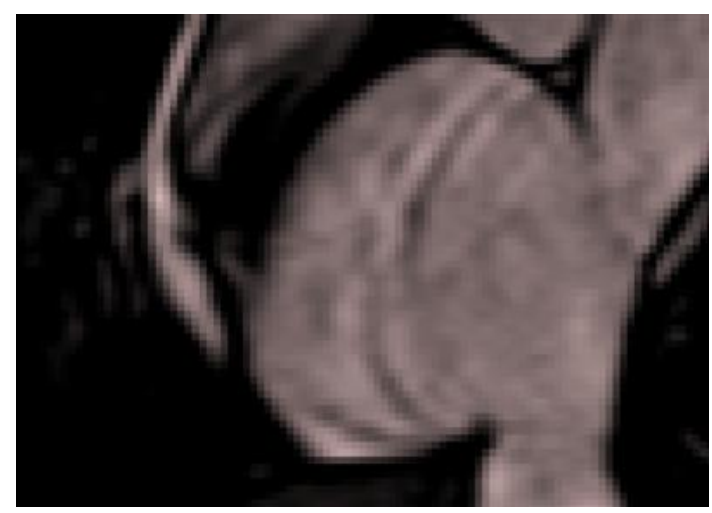

(e) $T=22$

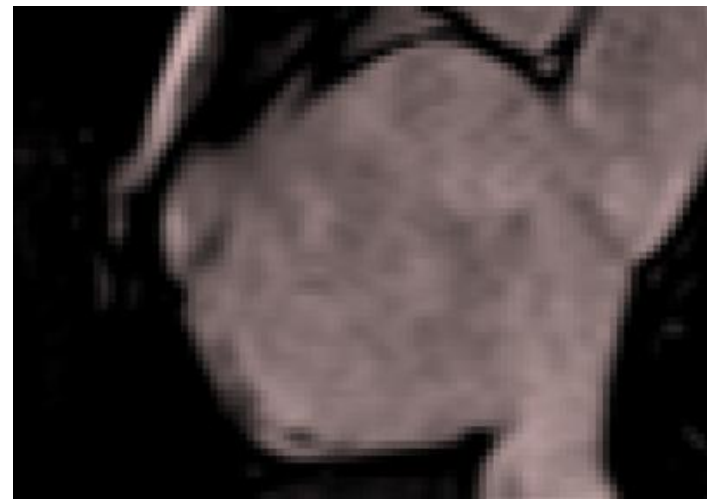

(b) $T=19$

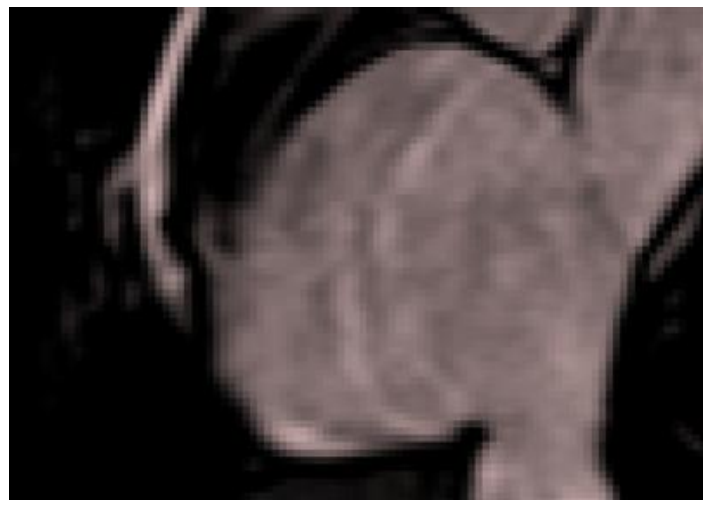

(d) $T=21$

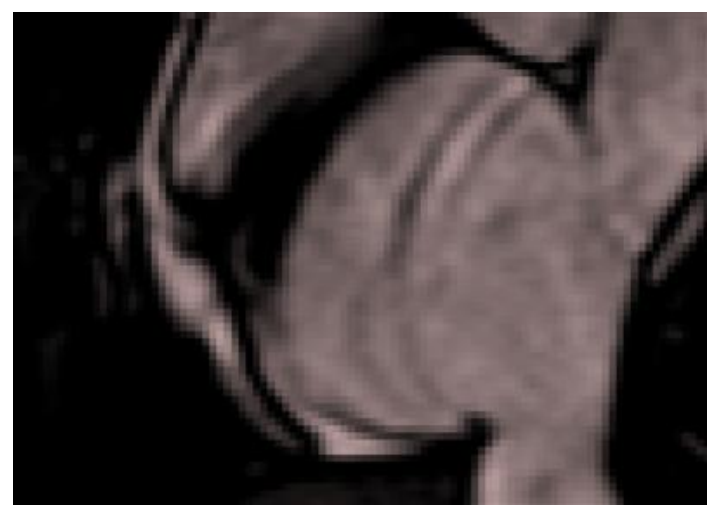

(f) $T=23$ 


\section{APPENDIX B. MAGNETIC RESONANCE IMAGE SCANS OF RIGHT ATRIUM (POST SEPTAL OCCLUDER INSERTION)}

Observation of blood leakage through occluder at ASD of heart

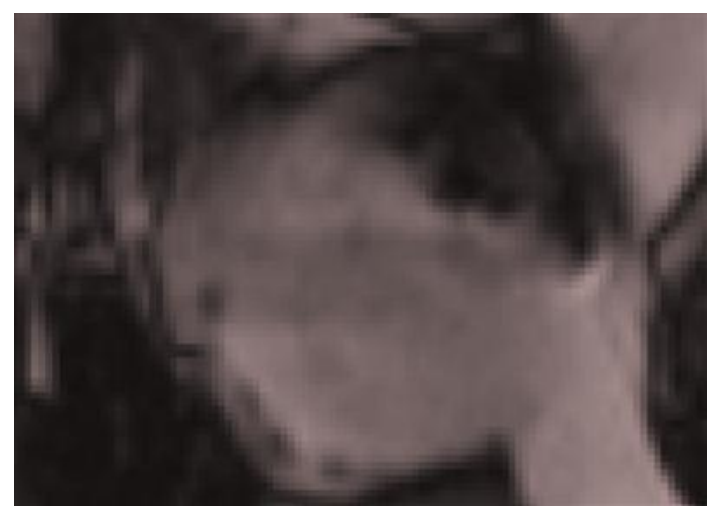

(g) $T=18$

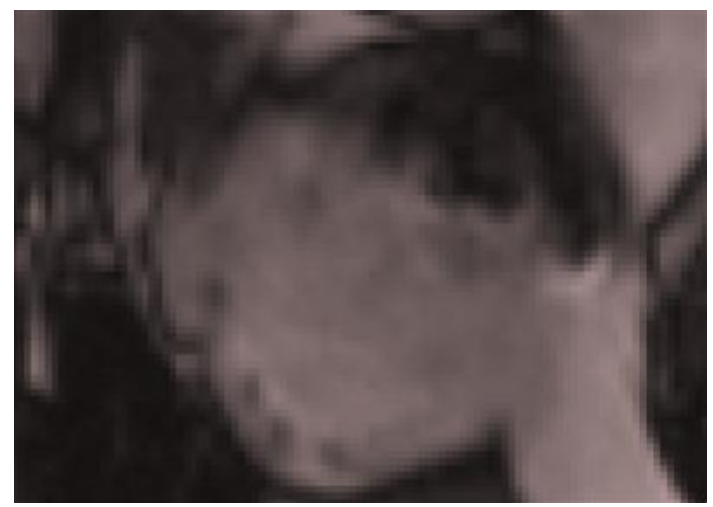

(i) $T=20$

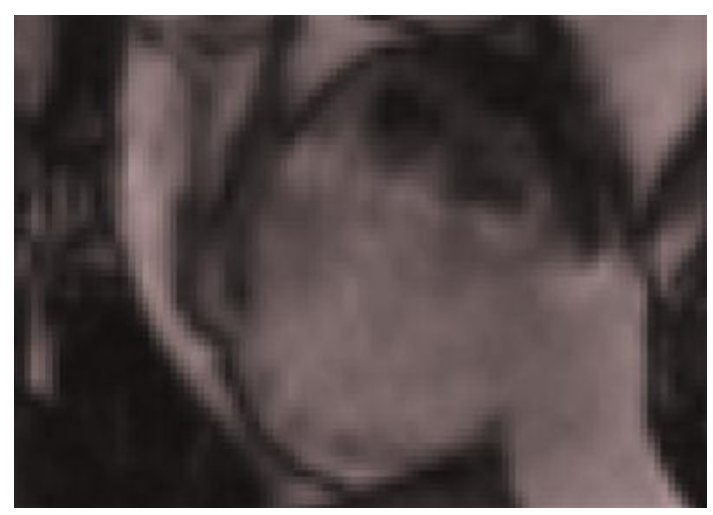

(k) $T=22$

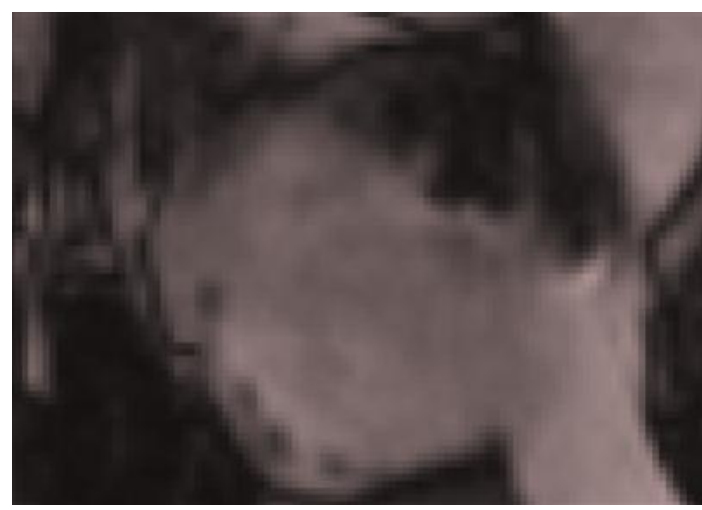

(h) $T=19$

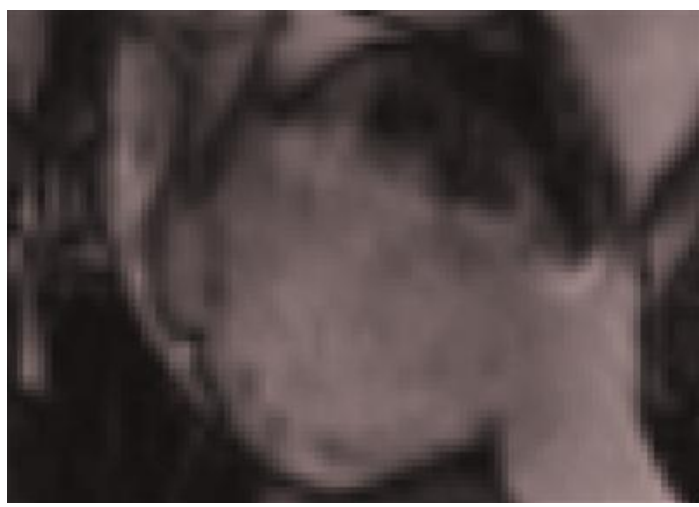

(j) $T=21$

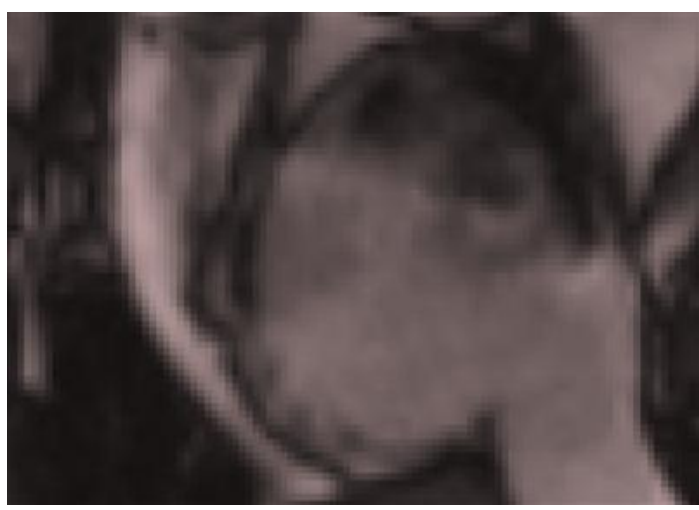

(l) $T=23$ 